\title{
5 LITERACIA EM SAÚdE MENTAL DOS JOVENS ESTUDANTES DE ENFERMAGEM NA INTEGRAÇÃO AO ENSINO SUPERIOR ${ }^{1}$
}

\author{
| Luís Loureiro; Paula Freitas² ${ }^{2}$
}

\section{RESUMO}

INTRODUÇÃO: A transição para o Ensino Superior e consequente processo de adaptação corresponde a um período crítico e complexo que acarreta mudanças significativas na vida dos jovens que podem vir a ter implicações na sua saúde e bem-estar e consequentemente no sucesso académico.

OBJETIVOS: Caracterizar a literacia em saúde mental associada à depressão e ansiedade; analisar comparativamente a literacia associada à depressão e ansiedade.

MÉTODOS: Estudo descritivo-correlacional, realizado a partir dos dados colhidos junto de uma amostra aleatória de 246 estudantes, inscritos no $1 .^{\circ}$ ano do curso de licenciatura em Enfermagem, tendo sido aplicado o Questionário de Avaliação da Literacia em Saúde Mental.

RESULTADOS: Os resultados revelam níveis modestos de literacia em saúde mental, com deficits mais acentuados no caso da depressão. Uma margem de jovens refere de modo explicito que não sabe e/ou não tem conhecimento em termos de primeira ajuda.

CONCLUSÕES: Torna-se necessário intervir precocemente, no sentido de aumentar os níveis de literacia de saúde mental dos estudantes. Estas intervenções, com foco no acolhimento e integração, podem contribuir para a adaptação e sucesso académico dos estudantes.

PALAVRAS-CHAVE: Literacia; Saúde mental; Depressão; Ansiedade

\section{RESUMEN}

"Alfabetización en salud mental de los jóvenes estudiantes de enfermería en la integración a la enseñanza superior”

INTRODUCCIÓN: La transición hacia la enseñanza superior y el consiguiente proceso de adaptación corresponde a un período crítico y complejo que conlleva cambios significativos en la vida de los jóvenes que pueden tener implicaciones en su salud y bienestar y consecuentemente en el éxito académico.

OBJETIVOS: Caracterizar la alfabetización en salud mental asociada a la depresión y ansiedad; analizar comparativamente la alfabetización asociada a la depresión y la ansiedad.

METODOLOGÍA: Estudio descriptivo-correlacional, realizado a partir de los datos recolectados en una muestra de 246 estudiantes, inscritos en el primer año del curso de licenciatura en Enfermería, habiendo sido aplicado el Cuestionario de Evaluación de la Alfabetización en Salud Mental.

RESULTADOS: Los resultados revelan niveles modestos de alfabetización en salud mental, con deficiencias más acentuadas en el caso de la depresión. Un margen de jóvenes señala que no sabe y / o no tiene conocimiento en este ámbito, como es el caso de las estrategias de primera ayuda.

CONCLUSIONES: Es necesario intervenir precozmente, en el sentido de aumentar sus niveles de alfabetización de salud mental de los estudiantes. Estas intervenciones, con foco en la acogida e integración, pueden contribuir a la adaptación y éxito académico de los estudiantes.

\section{DESCRIPTORES: Alfabetización; Salud mental; Depresión;} Ansiedad

\begin{abstract}
"Mental health literacy of young nursing students when accessing higher education"

INTRODUCTION: The transition to higher education and the consequent adaptation process corresponds to a critical and complex period that brings significant changes in the lives of young people that may have implications for their health and well-being and consequently academic success.

AIMS: To characterize the mental health literacy associated with depression and anxiety; analyze comparatively the literacy associated with depression and anxiety.

METHODS: Descriptive-correlational study, based on data collected from a random sample of 246 students, enrolled in the 1st year of a bachelor in Nursing, and the Questionnaire for Evaluation of Mental Health Literacy was applied.

RESULTS: The results reveal modest levels of mental health literacy, with a more pronounced deficiency in the case of depression. A margin of young people indicates that they do not know and/or do not have knowledge in this area, as is the case of first aid strategies.

CONCLUSIONS: It is necessary to intervene early in order to increase students' mental health literacy levels. These interventions, with a focus on reception and integration, can contribute to the students' academic adaptation and success.
\end{abstract}

\section{KEYWORDS: Literacy; Mental health; Depression; Anxiety}

Submetido em 30-09-2019

Aceite em 22-04-2020

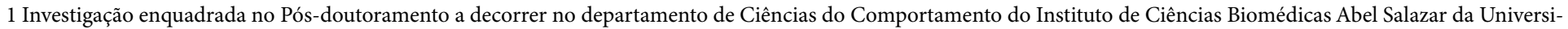
dade do Porto.

2 Doutor; Professor Adjunto na Escola Superior de Enfermagem de Coimbra, Rua 5 de Outubro (Polo B), Coimbra, Portugal, luisloureiro@esenfc.pt

3 Doutora; Professora Auxiliar na Universidade do Porto, Instituto de Ciências Biomédicas Abel Salazar, Porto, Portugal, pmfreitas@icbas.up.pt
}

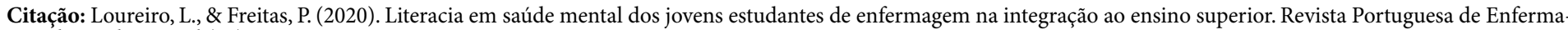
gem de Saúde Mental (24), 34-42. 


\section{INTRODUÇÃO}

A transição e consequente processo de adaptação ao ensino superior, corresponde a um período crítico que implica mudanças significativas na vida dos jovens (Almeida, 2014) aos níveis educativos, ecológicos e desenvolvimentais (Soares, Pereira e Canavarro, 2015).

A evidência produzida ao longo das últimas décadas, aponta para que a qualidade da adaptação seja considerada uma variável preditora do sucesso académico em todas as suas vertentes (Nogueira, 2017), e que envolve, não apenas os aspetos escolares, mas também os sociorrelacionais e psicológicos, tais como o desenvolvimento intelectual, a criação e manutenção de novas relações e vínculos interpessoais e sociais, e o desenvolvimento da identidade e da autonomia (Soares, Pinheiro e Canavarro, 2015; Van Rooij, Jansen \& van de Grift, 2018).

Neste sentido, a saúde mental e o bem-estar assumem um lugar de destaque, quer sejam perspetivados a partir do impacto que este processo de transição pode acarretar na saúde mental dos jovens, quer ainda por ser considerada uma condição que lhes permite adaptar-se e enfrentar com sucesso os desafios que lhes são colocados (Nogueira e Sequeira, 2018).

É também referido na literatura que nem todos os estudantes estão capacitados para enfrentar e gerir os desafios com sucesso, na medida em que muitos experienciam níveis elevados de stress, ansiedade e depressão (Araújo e Almeida, 2015; Nogueira, 2017). É comumente aceite que as dificuldades decorrentes do stress académico podem ter impacto no desencadear de problemas de saúde mental e que estes se podem agravar com o tempo $(\mathrm{Pu}-$ lido-Martos, Augusto-Landa \& Lopez-Zafra, 2012; Ribeiro et al., 2017). Acresce ainda o facto de uma franja dos jovens apresentar à partida problemas de saúde mental, alguns dos quais com perturbações psiquiátricas diagnosticadas (Silveira, Norton, Brandão e Roma-torres, 2011), que se podem agravar à medida que o percurso académico se desenrola, ao ponto de comprometer a sua autonomia, autoconfiança e perceção de competência pessoal e consequentemente o desempenho académico. Neste sentido as instituições de Ensino Superior devem potenciar continuamente os serviços de apoio e ajuda especializados, com o objetivo de maximizar o seu potencial de crescimento e desenvolvimento e minimizar os efeitos adversos das dificuldades de adaptação. Simultaneamente, poderão ser ainda utilizadas outras medidas/iniciativas centradas no acolhimento e integração dos estudantes e cujo foco se centre na transição e adaptação académica, de modo a potenciar, por exemplo o suporte social, a saúde mental e o bem-estar.

Poderemos destacar neste contexto as intervenções que ancoram no conceito de literacia em saúde mental (LSM) na medida em que as crenças e conhecimentos acerca dos problemas de saúde mental podem permitir aos jovens, reconhecer os problemas de saúde mental, geri-los no quotidiano, assim como tomar medidas preventivas adequadas (Jorm, 2014). Este conhecimento é voltado para a ação em prol da saúde mental e bem-estar do estudante e dos seus pares.

É de salientar que a evidência produzida no contexto Português ao nível da LSM de estudantes do ensino superior é reduzida, contudo os dados disponíveis relativamente às componentes de reconhecimento dos problemas de saúde mental (incluindo as perturbações mentais) e conhecimentos e competências para prestar a primeira ajuda apontam, por um lado, para que muitos jovens não reconheçam e não valorizem os problemas de saúde mental e em consequência deste facto, não procurem ajuda especializada ou atrasem a sua procura, e, por outro, apresentem deficits significativos em termos dos conhecimentos e competências para prestar primeira ajuda aos seus pares (Correia, 2012).

A escassez de dados relativamente à LSM dos estudantes do Ensino Superior, conjugados com aquela que a evidência acerca dos desafios que a transição e adaptação ao ensino superior implicam em termos da sua saúde mental e bem-estar dos jovens, estiveram na base de desenvolvimento do presente estudo e cujos objetivos são: a) caracterizar a literacia em saúde mental dos estudantes do $1 .^{\circ}$ ano do curso de licenciatura em Enfermagem em termos de depressão e ansiedade, nomeadamente ao nível do reconhecimento dos problemas, intenção de pedido de ajuda, conhecimentos sobre primeira ajuda e confiança para prestar primeira ajuda; b) analisar comparativamente a literacia associada aos problemas de saúde mental referidos.

\section{MÉTODOS}

O presente estudo, prossegue uma abordagem quantitativa de tipo descritivo-correlacional.

\section{Hipótese}

Os estudantes de Enfermagem apresentam, comparativamente, um nível diferenciado de LSM acerca da depressão e da ansiedade em termos de reconhecimento dos problemas, conhecimentos sobre primeira ajuda em saúde mental, intenção de pedido de ajuda e confiança para prestar ajuda. 


\section{Participantes}

O presente estudo foi realizado a partir dos dados colhidos junto de uma amostra aleatória simples de 246 estudantes, selecionados a partir dos estudantes inscritos no $1 .^{\circ}$ ano do curso de licenciatura em Enfermagem. A selecção foi realizada com recurso à aplicação random. org (https://www.random.org). Da lista inicial de 350 estudantes, foram selecionados de modo aleatório simples 250 estudantes (71\%), aos quais foram aplicados os questionários. Destes 250 questionários, 4 foram anulados por não se encontrarem devidamente preenchidos. O critério de inclusão foi: ser aluno do $1 .^{\circ}$ ano, inscrito pela primeira vez no curso de enfermagem.

A média das idades observada é de 18,77 anos (desvio padrão de 2,99 anos) e a mediana de 18 anos, sendo $41(16,70 \%)$ do sexo masculino e 205 (83,30\%) do sexo feminino.

\section{Instrumento de colheita de dados}

$\mathrm{O}$ instrumento de colheita de dados utilizado foi o Questionário de Avaliação da Literacia em Saúde Mental - QuALiSMental (Loureiro, 2015). Este questionário é composto por duas partes. A $1 .{ }^{\mathrm{a}}$ parte é constituída por questões de âmbito sociodemográfico, nomeadamente sexo, idade e habilitações literárias dos pais. A 2. ${ }^{a}$ parte compreende um conjunto de questões relacionadas com as diferentes componentes da literacia em saúde mental, nomeadamente: a) reconhecimento dos problemas de saúde mental (incluindo perturbações mentais) de modo a promover e facilitar a procura de ajuda e b) conhecimentos e competências para prestar apoio e primeira ajuda aos outros.

As questões de cada componente referida são precedidas por duas vinhetas, uma relatando um caso de uma jovem chamada Joana que apresenta sinais e sintomas de depressão, e outra chamada Rita que apresentava sinais e sintomas de quem sofria de problemas relacionados com ansiedade. Ambos os casos estão de acordo com os critérios da versão 5 do Manual de Diagnóstico e Estatística das Perturbações Mentais 5 - DSM-V da American Psychiatric Association (2014).

Depois de apresentadas as vinhetas, na componente de reconhecimento é colocada a seguinte questão: "Na tua opinião, o que é que se passa com a Joana/Rita? (Podes assinalar várias respostas). A possibilidade de respostas consiste em 17 itens, sendo que existem rótulos de problemas de saúde mental, bem como a possibilidade de escolher "não sei"; "não tem nada"; "tem um problema” ou "outro", devendo neste último caso, especificar qual.
Nos rótulos estão incluídos: "depressão"; "esquizofrenia”; "psicose"; "doença mental"; "bulimia”; "stress"; "esgotamento nervoso"; "abuso de substâncias (ex.: álcool)"; "crise própria da idade"; "problemas psicológicos/ mentais/ emocionais"; "anorexia"; "ansiedade" e "alcoolismo". A componente de conhecimentos e competências para prestar apoio e dar a primeira ajuda é constituída por 10 itens, sendo precedidos pela seguinte orientação: "São apresentadas de seguida diferentes opções que podias utilizar para ajudar a Joana/ Rita. Assinala para cada uma a tua opinião".

Os itens do questionário relativos a esta componente são: "ouvir os seus problemas de forma compreensiva"; "dizer-lhe com firmeza para andar para a frente"; "sugerir que procure ajuda de um profissional de saúde especializado"; "marcar uma consulta no médico de família com o seu conhecimento"; "perguntar se tem tendências suicidas"; "sugerir que beba uns copos para esquecer os problemas"; "reunir o grupo de amigos para a animar"; "não valorizar o seu problema, ignorando-a até que se sinta melhor"; "mantê-la ocupada para que não pense tanto nos seus problemas"; "incentivá-la a praticar exercício físico". O formato de resposta aos itens é "útil”, "prejudicial”, "não sei”).

Esta componente é ainda constituída por outras duas questões, nomeadamente:

a) "Se estivesses a viver atualmente uma situação como a da Joana/Rita, pedirias ajuda?". Na reposta os participantes podem assinalar "sim", "não" e "não sei"; b) "Em que medida te sente confiante para ajudar a Joana/Rita?". O formato de resposta é tipo Likert e varia de 1 (nada confiante) a 5 pontos (muitíssimo confiante).

A avaliação das características psicométricas do para a população portuguesa (Loureiro, 2015) revelou bons índices de fiabilidade, assim como validade de construto que atestam a sua utilização neste contexto, estando a tipologia e conteúdo do instrumento disponível em Loureiro (2015).

\section{Procedimentos ético-formais da colheita de dados}

Os dados foram recolhidos no mês de setembro de 2018 . Os questionários foram aplicados em contexto de sala de aula, em sessões coletivas, num período especificamente disponibilizado para o efeito. O tempo de resposta do questionário situou-se entre os 20 e os 30 minutos. A proposta do estudo foi submetida à Diretora da instituição onde foi realizado o estudo, tendo obtido parecer positivo e autorizada a sua realização. 
Dadas as caraterísticas da amostra, após serem informados sobre os objetivos do estudo, os participantes assinaram um termo de consentimento informado.

\section{Análise estatística}

Os dados foram analisados com recurso ao software IBM SPSS-24. Foram calculadas as estatísticas resumo adequadas (média, mediana e desvio padrão) para a idade e frequências absolutas e percentuais para a variável sexo. No caso do item relativo à confiança para prestar ajuda, também foram calculadas medidas resumo, nomeadamente de tendência central (média e mediana).

No que respeita ao estudo comparativo efetuado, e dado que a amostra, apesar do estímulo (vinheta) ser diferente, engloba as mesmas questões/itens, com os mesmos sujeitos a serem avaliados, o que tem subjacente emparelhamento, esse facto implicou a utilização de testes para grupos emparelhados. Dado o formato de resposta (qualitativo) dos itens, são utilizados testes não paramétricos, neste caso os testes estatísticos de $\mathrm{McNe}$ mar (tabelas de $2 \times 2$ ) e teste de Bowker (caso das tabelas de $3 \times 3$ ). Na questão "b" da última componente referida ("Em que medida se sente confiante para ajudar a Joana/ Rita?"), e dado o formato de resposta ser tipo Likert, utilizou-se o teste de sinais por postos de Wilcoxon.

Relativamente aos testes de McNemar e Bowker, dado o número de variáveis em estudo, o número de tabelas de dupla entrada (contingência) produzidas é extenso, pelo que se optou por resumir todos os resultados em tabelas que incluem as distribuições de frequências percentuais. O cálculo conjunto das frequências percentuais foi realizado com recurso à opção de tabelas customizadas do IBM SPSS-24.

O teste de Bowker, sendo um teste omnibus, implicou o cálculo adicional de testes post hoc com correção de Bonferroni, especificamente nos casos em que as diferenças encontradas foram estatisticamente significativas $(\mathrm{p}<0,05)$. Neste sentido, os procedimentos à posteriori implicaram o cálculo de três novas tabelas de dupla entrada (as restantes tabelas introduziam informação redundante e não forma utilizadas), que comparavam as respostas obtidas às categorias, duas a duas $(2 \times 2)$.

\section{RESULTADOS}

Os resultados são apresentados de acordo com a ordem das variáveis na hipótese submetida a teste: a) reconhecimento dos problemas, b) conhecimentos sobre primeira ajuda em saúde mental; c) intenção de pedido de ajuda e d) confiança para prestar ajuda e apoio.
Como se pode observar da Tabela 1 (apenas se apresentaram os rótulos com valores cuja frequência absoluta seja $\geq 1$ ), em termos de reconhecimento da depressão, $74,39 \%$ dos participantes assinalam o rótulo "depressão", seguido de "stress" (67,89\%), "ansiedade" (53,25\%), "esgotamento nervoso" (46,75\%) e "problemas psicológicos/mentais/emocionais" (50,00\%). No caso da vinheta $\mathrm{da}$ ansiedade, o rótulo assinalado em primeiro lugar foi a "ansiedade" (70,73\%), seguido de "problemas psicológicos/mentais/emocionais" (45,53\%) e "stress" (43,90\%). Em termos comparativos, os testes de McNemar revelam diferenças com significado estatístico nos rótulos “depressão $\left(\mathrm{x}^{2}=168,14 ; \mathrm{p}<0,001\right)$, "stress" $\left(\mathrm{x}^{2}=33,31\right.$; $\mathrm{p}<0,001)$, "esgotamento nervoso" $\left(\mathrm{x}^{2}=98,44 ; \mathrm{p}<0,001\right) \mathrm{e}$ ansiedade $\left(\mathrm{x}^{2}=15,57 ; \mathrm{p}<0,001\right)$.

Tabela 1 - Distribuição percentual das respostas assinaladas pelos participantes aos itens relativos ao reconhecimento, em função das vinhetas da depressão e ansiedade $(\mathrm{N}=246)$

\begin{tabular}{|c|c|c|c|c|c|}
\hline & & \multicolumn{3}{|c|}{ Vinheta da depressão } & \\
\hline \multicolumn{2}{|c|}{ Vinheta da ansiedade } & Sim & Não & Total & \\
\hline \multirow[t]{3}{*}{ Depressão } & Sim & 2,85 & 0,81 & 3,66 & \multirow{3}{*}{$168,14^{* * *}$} \\
\hline & Não & 71,54 & 24,80 & 96,34 & \\
\hline & Total & 74,39 & 25,61 & 100,00 & \\
\hline \multirow{3}{*}{$\begin{array}{l}\text { Doença } \\
\text { mental }\end{array}$} & Sim & 3,25 & 3,66 & 6,91 & \multirow{3}{*}{$(\dagger) \mathrm{ns}$} \\
\hline & Não & 5,28 & 87,80 & 93,09 & \\
\hline & Total & 8,54 & 91,46 & 100,00 & \\
\hline \multirow[t]{3}{*}{ Stresse } & Sim & 35,37 & 8,54 & 43,90 & \multirow{3}{*}{$33,31^{* * *}$} \\
\hline & Não & 32,52 & 23,58 & 56,10 & \\
\hline & Total & 67,89 & 32,11 & 100,00 & \\
\hline \multirow{3}{*}{$\begin{array}{l}\text { Esgotamento } \\
\text { nervoso }\end{array}$} & Sim & 2,44 & 1,22 & 3,66 & \multirow{3}{*}{$98,44^{* * *}$} \\
\hline & Não & 44,31 & 52,03 & 96,34 & \\
\hline & Total & 46,75 & 53,25 & 100,00 & \\
\hline \multirow[t]{3}{*}{ Crise da idade } & Sim & 1,22 & 3,27 & 4,49 & \multirow{3}{*}{$(\dagger) \mathrm{ns}$} \\
\hline & Não & 6,94 & 88,57 & 95,51 & \\
\hline & Total & 8,16 & 91,84 & 100,00 & \\
\hline \multirow{3}{*}{$\begin{array}{l}\text { Problemas } \\
\text { psicológicos/ } \\
\text { mentais/emo- } \\
\text { cionais }\end{array}$} & Sim & 30,49 & 15,04 & 45,53 & \multirow{3}{*}{$1,18 \mathrm{~ns}$} \\
\hline & Não & 19,51 & 34,96 & 54,47 & \\
\hline & Total & 50,00 & 50,00 & 100,00 & \\
\hline \multirow[t]{3}{*}{ Ansiedade } & Sim & 42,68 & 28,05 & 70,73 & \multirow{3}{*}{$15,57^{\star * *}$} \\
\hline & Não & 10,57 & 18,70 & 29,27 & \\
\hline & Total & 53,25 & 46,75 & 100,00 & \\
\hline
\end{tabular}

$\dagger$ Utilizada distribuição binomial; ns = não significativo; ${ }^{* * *} \mathrm{p}<0,001$

Ao nível dos conhecimentos acerca das estratégias para prestar primeira ajuda aos outros (Tabela 2), os resultados indicam que a quase totalidade dos jovens (> 99,00\%) percecionam como "útil” a escuta compreensiva para ambos problemas de saúde descritos nas vinhetas. 
No caso de outras estratégias que são consideradas adequadas, tais como "sugerir que procure ajuda de um profissional", "marcar uma consulta no médico de família", "incentivá-la para que pratique exercício físico", ainda que uma percentagem muito substancial dos jovens as percecionem como uteis para a depressão, respetivamente $85,71 \%, 71,72 \%$ e $80,08 \%$, esse valor diminui comparativamente para os mesmos itens, na ansiedade (57,96\%, $41,39 \%$ e $74,39 \%)$, com destaque para as duas primeiras estratégias.

Nas estratégias consideradas ineficazes e desajustadas em termos de primeira ajuda, nomeadamente "não valorizar o seu problema, ignorando-a até que se sinta melhor", "mantê-la ocupada para que não pense tanto nos problemas", "dizer-lhe com firmeza para andar para a frente" e "sugerir que beba uns copos para esquecer os problemas" os jovens apresentam um perfil diferenciado de respostas. A quase totalidade considera consumo de álcool como "prejudicial", respetivamente 92,24\% na depressão e $89,80 \%$ na ansiedade. Contudo, nas outras estratégias, a opção "não sei” apresenta percentagens relativamente elevadas, especificamente no caso de "dizerlhe com firmeza para andar para a frente" $(43,28 \%$ na depressão e 36,97\% na ansiedade).

Também a estratégia "mantê-la a pessoa ocupada para que não pense tanto nos problemas" é considerada estratégia útil em ambos os casos por 74,29\% na depressão e $66,12 \%$ na ansiedade.

Um dos itens com resultados que carecem de reflexão em termos de primeira ajuda, especificamente no caso da vinheta da depressão, diz respeito ao questionar a pessoa se tem tendências suicidas. Como podemos observar da Tabela 2, 48,16\% afirmam que é prejudicial e $31,84 \%$ afirmam que não sabem.

Comparadas as estratégias em função das duas vinhetas, o teste de Bowker revela diferenças com significado estatístico em quatro estratégias: "sugerir que procure ajuda de um profissional de saúde" $\left(\mathrm{x}^{2}=57,95\right.$; $\mathrm{p}<0,001)$; "marcar uma consulta no médico de família" $\left(x^{2}=59,89 ; \mathrm{p}<0,001\right)$; "perguntar se tem tendências suicidas $\left(\mathrm{x}^{2}=32,27 ; \mathrm{p}<0,001\right)$; "reunir o grupo de amigos para a animar" $\left(x^{2}=10,57 ; p<0,05\right)$ e "mantê-la ocupada para que não pense tanto nos problemas" ( $\left.x^{2}=9,37 ; p<0,05\right)$.

Nos itens referidos anteriormente (Tabela 2), os procedimentos post hoc apenas revelaram diferenças quando comparadas as categorias "útil" e "não sei” nos casos em que a mudança é estatisticamente significativa ao nível de 0,001 .
Neste sentido, os "reunir o grupo de amigos para a animar" e "mantê-la ocupada para que não pense tanto nos problemas", apesar de inicialmente terem significado estatístico, não vieram a relevar diferenças dada a aplicação da correção não revelou diferenças em nenhum dos grupos. Os valores das distribuições percentuais e que incluem os testes post hoc são apresentados na Tabela 3. Ainda na Tabela 2, podemos observar no que respeita à intenção de pedido de ajuda

em situação idêntica à referida nas vinhetas, que as mudanças são estatisticamente quando se passa da depressão para a ansiedade $\left(\mathrm{x}_{2}=24,52 ; \mathrm{p}<0,001\right)$.

Quando questionados acerca da sua confiança para prestar ajuda e apoio à pessoa que estava nas situações descritas nas vinhetas (Tabela 2), o teste de sinais por postos de Wilcoxon indicou que as diferenças não têm significado estatístico $(\mathrm{z}=-0,76 ; \mathrm{p}>0,05)$. As médias são respetivamente de 2,87 pontos no caso da depressão (mediana $=3,00$ pontos) e 2,75 pontos na ansiedade (mediana $=3,00$ pontos).

Tabela 2 - Distribuição percentual das respostas assinaladas pelos participantes aos itens sobre conhecimentos sobre primeira ajuda em saúde mental e intenção de procura de ajuda para as vinhetas da depressão e ansiedade $(\mathrm{N}=246)$

\begin{tabular}{|c|c|c|c|c|c|c|}
\hline & & \multicolumn{4}{|c|}{ Vinheta da depressão } & \\
\hline \multicolumn{2}{|c|}{ Vinheta da ansiedade } & A & B & $\mathrm{C}$ & Total & $\mathrm{x}^{2}(\dagger)$ \\
\hline \multirow{4}{*}{$\begin{array}{l}\text { Ouvir os seus } \\
\text { problemas de } \\
\text { forma com- } \\
\text { preensiva }\end{array}$} & A & 98,80 & --- & 0,80 & 99,60 & \multirow{4}{*}{$(\dagger \dagger) \mathrm{ns}$} \\
\hline & B & --- & --- & --- & --- & \\
\hline & $\mathrm{C}$ & 0,40 & --- & 0,00 & 0,40 & \\
\hline & Total & 99,20 & --- & 0,81 & 100,00 & \\
\hline \multirow{4}{*}{$\begin{array}{l}\text { Dizer-lhe com } \\
\text { firmeza para } \\
\text { andar para a } \\
\text { frente }\end{array}$} & A & 16,81 & 5,04 & 12,18 & 34,03 & \multirow{4}{*}{$4,65 \mathrm{~ns}$} \\
\hline & B & 2,52 & 17,65 & 8,82 & 28,99 & \\
\hline & $\mathrm{C}$ & 8,40 & 6,30 & 22,27 & 36,97 & \\
\hline & Total & 27,73 & 28,99 & 43,28 & 100,00 & \\
\hline \multirow{4}{*}{$\begin{array}{l}\text { Sugerir que } \\
\text { procure } \\
\text { ajuda de um } \\
\text { profissional de } \\
\text { saúde }(\dagger \dagger \dagger)\end{array}$} & A & 55,51 & --- & 2,45 & 57,96 & \multirow{4}{*}{$57,95^{* * *}$} \\
\hline & $\mathrm{B}$ & 2,45 & 0,82 & 0,82 & 4,08 & \\
\hline & $\mathrm{C}$ & 27,76 & 0,82 & 9,39 & 37,96 & \\
\hline & Total & 85,71 & 1,63 & 12,65 & 100,00 & \\
\hline \multirow{4}{*}{$\begin{array}{l}\text { Marcar uma } \\
\text { consulta no } \\
\text { médico de } \\
\text { família }(\dagger \dagger \dagger)\end{array}$} & $\mathrm{A}$ & 37,70 & --- & 3,69 & 41,39 & \multirow{4}{*}{$59,89^{* * *}$} \\
\hline & B & 1,64 & 3,28 & 1,23 & 6,15 & \\
\hline & $\mathrm{C}$ & 32,38 & 0,82 & 19,26 & 52,46 & \\
\hline & Total & 71,72 & 4,10 & 24,18 & 100,00 & \\
\hline \multirow{4}{*}{$\begin{array}{l}\text { Perguntar se } \\
\text { tem tendên- } \\
\text { cias } \\
\text { suicidas }(\dagger \dagger \dagger)\end{array}$} & $\mathrm{A}$ & 7,35 & --- & 0,41 & 7,76 & \multirow{4}{*}{$32,27^{* * *}$} \\
\hline & B & 2,04 & 42,45 & 11,02 & 55,51 & \\
\hline & $\mathrm{C}$ & 10,61 & 5,71 & 20,41 & 36,73 & \\
\hline & Total & 20,00 & 48,16 & 31,84 & 100,00 & \\
\hline \multirow{4}{*}{$\begin{array}{l}\text { Sugerir que } \\
\text { beba uns } \\
\text { copos para } \\
\text { esquecer os } \\
\text { problemas }\end{array}$} & $\mathrm{A}$ & --- & 2,04 & 41 & 2,45 & \multirow{4}{*}{$2,76 \mathrm{~ns}$} \\
\hline & B & ,82 & 85,71 & 3,27 & 89,80 & \\
\hline & $\mathrm{C}$ & $-\cdots$ & 4,49 & 3,27 & 7,76 & \\
\hline & Total & 82 & 92,24 & 6,94 & 100,00 & \\
\hline
\end{tabular}




\begin{tabular}{|c|c|c|c|c|c|c|}
\hline \multirow{4}{*}{$\begin{array}{l}\text { Reunir o } \\
\text { grupo de } \\
\text { amigos para a } \\
\text { animar }\end{array}$} & $\mathrm{A}$ & 73,36 & 1,64 & 6,56 & 81,56 & \multirow{4}{*}{$10,57^{\star}$} \\
\hline & B & 5,33 & 0,41 & 1,64 & 7,38 & \\
\hline & $\mathrm{C}$ & 9,84 & --- & 1,23 & 11,07 & \\
\hline & Total & 88,52 & 2,05 & 9,43 & 100,00 & \\
\hline \multirow{4}{*}{$\begin{array}{l}\text { Não valorizar } \\
\text { o seu prob- } \\
\text { lema, ignoran- } \\
\text { do-a até }\end{array}$} & A & --- & ,82 & --- & ,82 & \multirow{4}{*}{$3,57 \mathrm{~ns}$} \\
\hline & B & ,82 & 92,24 & 1,63 & 94,69 & \\
\hline & $\mathrm{C}$ & ,41 & 4,08 & --- & 4,49 & \\
\hline & Total & 1,22 & 97,14 & 1,63 & 100,00 & \\
\hline \multirow{4}{*}{$\begin{array}{l}\text { Mantê-la } \\
\text { ocupada para } \\
\text { que não pense } \\
\text { tanto }\end{array}$} & A & 57,55 & 0,82 & 7,76 & 66,12 & \multirow{4}{*}{$9,37^{\star}$} \\
\hline & $\mathrm{B}$ & 4,90 & 3,67 & 1,22 & 9,80 & \\
\hline & $\mathrm{C}$ & 11,84 & 1,63 & 10,61 & 24,08 & \\
\hline & Total & 74,29 & 6,12 & 19,59 & 100,00 & \\
\hline \multirow{4}{*}{$\begin{array}{l}\text { Incentivá-la a } \\
\text { praticar exer- } \\
\text { cício físico }\end{array}$} & $\mathrm{A}$ & 67,89 & --- & 6,50 & 74,39 & \multirow{4}{*}{--- } \\
\hline & $\mathrm{B}$ & 0,41 & --- & --- & 0,41 & \\
\hline & $\mathrm{C}$ & 11,79 & --- & 13,41 & 25,20 & \\
\hline & Total & 80,08 & --- & 19,92 & 100,00 & \\
\hline & & Sim & Não & $\begin{array}{c}\text { Não } \\
\text { sei }\end{array}$ & Total & $\mathrm{x}^{2}$ \\
\hline \multirow{4}{*}{$\begin{array}{l}\text { Se estivesses } \\
\text { a viver uma } \\
\text { situação idên- } \\
\text { tica, pedirias } \\
\text { ajuda? }(\dagger \dagger \dagger)\end{array}$} & Sim & 42,28 & 1,22 & 6,91 & 50,41 & \multirow{4}{*}{$24,52^{\star * *}$} \\
\hline & Não & 2,44 & 1,63 & 6,10 & 10,16 & \\
\hline & $\begin{array}{l}\text { Não } \\
\text { sei }\end{array}$ & 19,92 & 1,22 & 18,29 & 39,43 & \\
\hline & Total & 64,63 & 4,07 & 31,30 & 100,00 & \\
\hline \multirow{2}{*}{\multicolumn{2}{|c|}{$\begin{array}{l}\text { Confiança para prestar } \\
\text { ajuda }\end{array}$}} & Média & $\begin{array}{c}\text { Medi- } \\
\text { ana }\end{array}$ & Média & $\begin{array}{c}\text { Medi- } \\
\text { ana }\end{array}$ & $z \S$ \\
\hline & & 2,87 & 3,00 & 2,75 & 3,00 & $0,76 \mathrm{~ns}$ \\
\hline
\end{tabular}

$\mathrm{A}=$ útil; $\mathrm{B}=$ prejudicial; $\mathrm{C}=$ Não sei; $(\dagger)$ Estatística do teste de Bowker; (††) Teste de McNemar com distribuição binomial; $n s=$ não significativo; ${ }^{*} \mathrm{p}<0,05 ;{ }^{* * *} \mathrm{p}<0,001$; $\S \mathrm{z}$ do teste de sinais por postos de Wilcoxon; $(\dagger \dagger \dagger)$ itens submetidos a testes post hoc. Neste caso as matrizes cuja diferença apresentou significado estatístico foram as células AC ("útil" versus "não sei") em $2 \times 2$ com p $<0,01$ (consultar batela 3).

Relativamente aos testes post hoc e como referido anteriormente, apenas foram encontradas diferenças à posteriori nas matrizes em quatro itens ("sugerir que procure ajuda de um profissional de saúde"; "marcar uma consulta no médico de família"; "perguntar se tem tendências suicidas e "intenção de pedido de ajuda), sendo os resultados apresentados na Tabela 3.

No caso, "sugerir que procure ajuda de um profissional de saúde", o número de participantes que considera "útil" essa estratégia para a depressão são $87,55 \%$, valor que se situa em $60,94 \%$ na ansiedade. Na depressão, $12,45 \%$ refere não saber, enquanto na ansiedade esse valor é de $39,06 \%$. É de salientar que nesta matriz são apenas considerados 234 sujeitos, dado que os que respondem "prejudicial” são excluídos no cálculo do teste.

Na estratégia "marcar uma consulta no médico de família”, enquanto na depressão, 75,33\% considera "útil” a estratégia, na ansiedade esse valor é inferior (44,49\%), e em termos dos que afirmam não saber, esse valor é de 24,67\% na depressão, e de 55,51\% na ansiedade.
O tamanho do grupo considerado nesta análise é $\mathrm{n}=229$. Por último, a estratégia "perguntar se tem tendências suicidas" ( $n=96$ ), na depressão $46,90 \%$ considera útil”, enquanto na ansiedade o valor é menor (20,00\%), já no que respeita aos que afirmam "não sei", passa de 53,68\% na depressão para $80,00 \%$ na ansiedade.

No que respeita à intenção de pedido de ajuda os testes post hoc indicam duas matrizes onde se observam mudanças significativas, nomeadamente a matriz "AC", que considera "sim" e "não sei". Neste caso $(n=215)$ e no que respeita à depressão, 56,28\% assinalou que procuraria ajuda enquanto na ansiedade aumenta para $71,16 \%$. A ambivalência na procura de ajuda para a vinheta da depressão (assinalam 28,84\%), diminui na ansiedade, onde apenas $28,80 \%$ assinala a opção "não sei".

Quando comparada a matriz com as categorias "BC" (considerando $n=67$ ), isto é, para os formatos de resposta "não" e "não sei”, verifica-se que na depressão, $10,45 \%$ afirmam que não procurariam ajuda e $89,55 \%$ não sabem se o fariam, contudo na ansiedade, $28,36 \%$ não procurariam ajuda, enquanto os que têm dúvidas aumenta para $71,64 \%$.

Tabela 3 - Distribuição percentual das respostas assinaladas pelos participantes aos itens sobre conhecimentos sobre primeira ajuda em saúde mental e intenção de procura de ajuda para as vinhetas da depressão e ansiedade

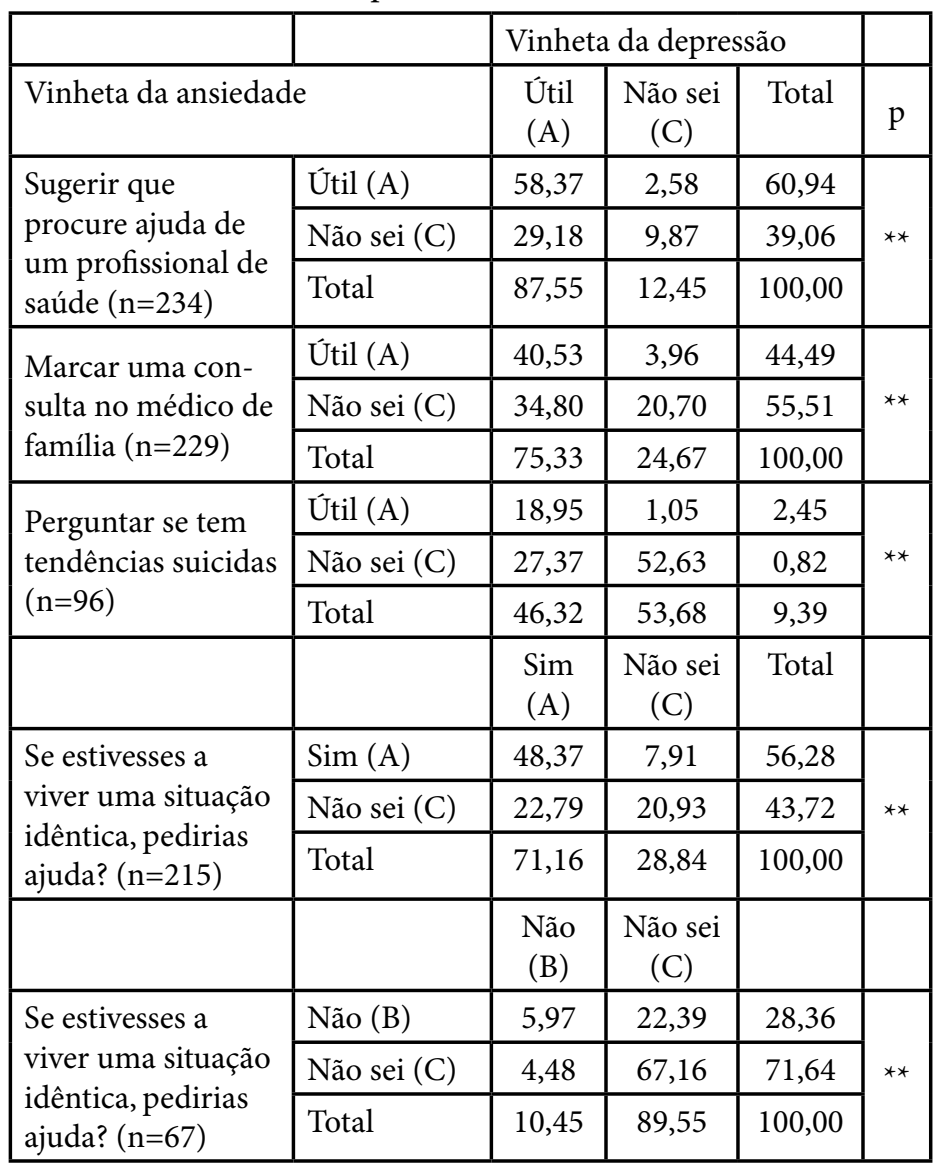

${ }^{* *} \mathrm{p}<0,01$ 


\section{DISCUSSÃO}

Em termos de reconhecimento dos problemas de saúde mental (e perturbações mentais) de modo a promover e facilitar a procura de ajuda, os resultados mostramse, em parte, satisfatórios e estão em linha com os obtidos noutros estudos (Correia, 2012; Jorm, 2014). É de salientar que nesta componente não se pretende avaliar a capacidade dos jovens em fazer diagnósticos em saúde mental, mas sim de problematizar e reconhecer os sinais de modo ajustado e valorizar os sintomas atribuindolhe neste sentido um rótulo ajustado ao problema descrito (Loureiro, 2014). Como se pode observar, $3 / 4$ da amostra assinala corretamente o rótulo "depressão" e "ansiedade" nas respetivas vinhetas sendo que 50,00\% dos participantes considera ambos os problemas descritos nas vinhetas como problemas psicológicos/mentais/ emocionais.

Contudo, no caso da depressão, tal como acontece noutros estudos (Correia, 2012; Loureiro, 2016), aproximadamente metade da amostra assinala o rótulo "esgotamento nervoso". Este rótulo é inespecífico e não se refere a um problema em particular, servindo mais para caraterizar qualquer tipo de mudança na saúde mental das pessoas. Acresce ainda uma aparente confusão dos sintomas descritos com problemas de "stress" e "ansiedade", o que pode indiciar um aparente desconhecimento do que significam e implicam estes problemas.

Comparativamente, verificamos que na vinheta da ansiedade tal questão não se coloca, pois assinalam maioritariamente os rótulos adequados ("stress"/"ansiedade" /" problemas psicológicos/mentais/emocionais”).

Como se pôde observar, uma margem reduzida de participantes assinala as situações como sendo doenças mentais $(<10,00 \%$ em ambos). Estes resultados podem indiciar que os jovens estudantes sabem em certa medida que para se falar em doença/perturbação mental implica a existência de um diagnóstico e neste sentido podemos verificar que a valorização é feita ao conceito de problema de saúde mental. É de ressaltar que o conteúdo das vinhetas não refere qualquer tipo de diagnóstico ou consulta especializada em saúde mental.

No que respeita às estratégias para prestar primeira ajuda aos outros, os estudos (Jorm, 2014; Correia, 2012; Loureiro, Sousa e Gomes, 2014; Loureiro, 2016), são unanimes em mostrar que a escuta compreensiva é eleita como a estratégia com maior utilidade na abordagem à pessoa que está a sofrer de um problema relacionado com a sua saúde mental.
Este dado é positivo e está em consonância com as propostas, por exemplo, da intervenção ao nível dos primeiros socorros em saúde mental (Loureiro, 2014; Loureiro e Sousa, 2019; Loureiro e Costa, 2019).

A estratégia de perguntar se tem pensamentos suicidas é vista, por cerca de metade da amostra como prejudicial, sendo que ainda cerca de $1 / 3$ afirma não saber da sua adequabilidade à situação. Esta relutância é explicada em parte pela crença de que este tipo de questões coloca a ideia de suicídio na pessoa.

Observa-se que uma margem substancial de jovens afirma o seu desconhecimento sobre algumas estratégias consideradas desadequadas, tanto para a depressão como para a ansiedade, tais como "dizer-lhe com firmeza para andar para a frente" e "mantê-la ocupada para que não pense tanto", assumindo que não sabem do efeito que este tipo de estratégia pode provocar na pessoa, tal como reportado noutros estudos (Correia, 2012; Jorm, 2014; Loureiro e Sousa; 2019; Loureiro e Costa, 2019).

Quando questionados sobre a intenção de procura de ajuda, caso vivenciassem uma situação semelhante à descrita nas vinhetas, aproximadamente $60 \%$ responde afirmativamente no caso da depressão e $50 \%$ na ansiedade. A evidência tem mostrado a este respeito que os pares (amigos, colegas), são eleitos como elementos essenciais no suporte à procura de ajuda em saúde mental e este facto é particularmente importante na adolescência e juventude, podendo por isso evitar-se o agudizar das situações.

Ao nível da confiança para prestar ajuda a alguém que esteja a sofrer de um problema idêntico ao descrito nas vinhetas, com valores estão também eles em consonância com os encontrados noutros estudos realizados com amostras de estudantes de Enfermagem (Correia, 2012; Loureiro e Sousa, 2019; Loureiro e Costa, 2019) ainda que frequentando anos letivos diferenciados. Mesmo que os desenhos sejam de cariz experimental (nível IV), no momento prévio à intervenção os estudantes sentem-se confiantes. Estes resultados merecem uma leitura cautelosa, até porque apesar de referirem que se sentem confiantes, nas estratégias de primeira ajuda uma margem considerável como se referiu não sabe da adequabilidade e utilidade das estratégias.

Se praticamente todos elegem como "útil" "ouvir os seus problemas de forma compreensiva", não sabemos que estratégias utilizariam para abordar a pessoa (Loureiro, Sousa e Gomes, 2014). A prática indica que se a abordagem à pessoa que está em sofrimento não for planeada, o dano causado poderá ser maior no sentido de poder levar a pessoa à escusa da procura de ajuda e a opção pela contínua ocultação dos sintomas (Loureiro, 2014). 
Sabemos que muitos jovens não reconhecem os problemas de saúde mental e consequentemente, nas situações que podem envolver sofrimento psicológico ou adoecer mental, não procuram ajuda, mesmo quando as instituições disponibilizam serviços de apoio social e psicológico, tão pouco são capazes de prestar primeira ajuda aos seus pares. $\mathrm{O}$ agudizar destes problemas pode levar ao comprometimento da sua saúde e bem-estar, ao ponto de levar ao abandono do curso e a projetos de futuro, ou ainda à ocorrência de outros comportamentos como o consumo de álcool e outras substâncias psicoativas. Concomitantemente a evidência mostra que uma boa rede de suporte social e familiar reduz o impacto dos acontecimentos stressantes e pode promover a procura de ajuda e maximização do potencial de desenvolvimento dos estudantes. Ora, em termos de estudantes de ensino superior, este problema pode agudizar-se ainda mais quando uma parte substancial dos estudantes estão deslocados e isso implica claramente um afastamento da sua rede de suporte social e familiar, ainda que se criem novos vínculos e relações pessoais e interpessoais, sabemos que os jovens privilegiam e valorizam a ajuda informal, nomeadamente dos pais e amigos.

Em termos globais, os resultados constituem um desafio face às necessidades que decorrem da missão social das instituições de ensino superior, especificamente ao nível do acolhimento e integração dos estudantes do $1 .^{\circ}$ ano. No caso dos estudantes de Enfermagem acrescem ainda outros desafios e que decorrem, por exemplo, da exposição ao longo do curso, logo a começar no primeiro ano, a um conjunto de stressores com fortes implicações na sua saúde e bem-estar, nomeadamente de âmbito académico, clínico e, inclusive, de âmbito pessoal.

Neste sentido, intervir precocemente pode contribuir para evitar o desenvolvimento e agravamento dos problemas de saúde mental que podem comprometer o seu sucesso académico. Nesta área, a evidência científica acerca da saúde mental e vulnerabilidade aponta para dados preocupantes relativamente à sua saúde mental (Sequeira, Carvalho, Borges e Sousa, 2013).

\section{CONCLUSÕES}

Os resultados permitem-nos concluir que o nível de literacia em saúde mental dos jovens estudantes que ingressam no $1 .^{\circ}$ ano do curso de Enfermagem revela deficits no reconhecimento dos problemas de saúde mental e nas estratégias de prestação de ajuda e apoio, com maior enfâse no caso da depressão.
É nosso entendimento que o desconhecimento relativo à saúde mental poderá ter implicações, entre outros, na procura de ajuda em saúde mental, especificamente nas situações queenvolvem vulnerabilidade e adoecermental. No contexto do curso de Enfermagem, que envolve uma forte componente humana, centrada na escuta e na relação, peças fundamentais do cuidar, as intervenções conducentes à promoção da saúde mental e bem-estar dos estudantes são de valorizar desde o início do percurso académico, centrando-se, por exemplo, no incremento da LSM dos estudantes em todas as suas componentes. Uma das formas de intervir poderá ser através da implementação do programa educacional de primeira ajuda em saúde mental (PASM), na medida em que este programa promove o conhecimento em saúde mental e potencia as estratégias de gestão da saúde mental no quotidiano.

Como o PASM é voltado para a acção em prol da saúde mental dos indivíduos e daqueles que o rodeiam, esta acção deve incluir todos os atores da comunidade educativa, incluindo pessoal docente e não docente das instituições de ensino superior.

\section{IMPLICAÇÕES PARA A PRÁTICA CLÍNICA}

Estes resultados constituem um incentivo e um alerta para instituições de Ensino Superior da Enfermagem no sentido de implementarem programas educacionais promotores da saúde mental dos jovens estudantes, centrados nos problemas e desafios com que se debatem em termos de saúde mental e bem-estar.

\section{REFERÊNCIAS BIBLIOGRÁFICAS}

Almeida, J. (2014). A saúde mental global, a depressão, a ansiedade e os comportamentos de risco nos estudantes do ensino superior: estudo de prevalência e correlação (Tese de Doutoramento). Universidade Nova de Lisboa, Portugal.

American Psychiatric Association. (2014). DSM-5 Manual de diagnóstico e estatística das perturbações mentais [Diagnostic and Statistical Manual of Mental Disorders (DSM-V)] (5th ed.). Porto Alegre: Artmed Editora.

Araújo, A. M., \& Almeida, L. S. (2015). Adaptação ao ensino superior: o papel moderador das expectativas acadêmicas. Lumen: Educare, 1(1), 13-31. Doi: 10.19141/2447-5432/lumen.v1.n1.p.13-32 
Correia, S. I. P. (2012). Construção e Validação de um Instrumento de Avaliação de Literacia em Saúde Mental para Adolescentes e Jovens (Dissertação de Mestrado). Escola Superior de Enfermagem de Coimbra, Portugal.

Jorm, A. F. (2014). Mental Health Literacy: Promoting Public Action to Reduce Mental Health Problems. In Literacia em Saúde Mental - Capacitar as Pessoas e as Comunidades para Agir (Vol. 8, pp. 27-39). Coimbra: Unidade de Investigação em Ciências da Saúde: Enfermagem.

Loureiro, L. (2015). Questionário de avaliação da literacia em saúde mental - QuALiSMental: estudo das propriedades psicométricas. Revista de Enfermagem Referência, IV(4), 79-88. Doi: 10.12707/RIV 14031

Loureiro, L. M. (2016). Literacia em saúde mental acerca da depressão e abuso de álcool de adolescentes e jovens Portugueses. Revista Portuguesa de Investigação Comportamental e Social, 2(2), 2-11. Doi: 10.7342/ismt. rpics.2016.2.2.33

Loureiro, L. M. (2014). Primeira Ajuda em Saúde Mental. Coimbra: Unidade de Investigação em Ciências da Saúde: Enfermagem.

Loureiro, L. M., \& Sousa, C. F. (2019). Programa de Primeiros Socorros em Saúde Mental: Estudo piloto. Revista Portuguesa De Investigação Comportamental e Social, 5(1), 72-86. Doi: 10.31211/rpics.2019.5.1.108

Loureiro, L., \& Costa, L. (2019). Avaliação do programa de Primeiros Socorros em Saúde Mental em estudantes de licenciatura em enfermagem. Revista de Enfermagem Referência, IV(20), 9-18. Doi: 10.12707/RIV18087

Loureiro, L., Sousa, C., \& Gomes, S. (2014). Primeira ajuda em saúde mental: Pressupostos e linhas orientadoras de acção. In Literacia em Saúde Mental - Capacitar as Pessoas e as Comunidades para Agir (Vol. 8, pp. 63-77). Coimbra, Portugal: Unidade de Investigação em Ciências da Saúde: Enfermagem.

Nogueira, M. (2017). Saúde mental em estudantes do ensino superior: fatores protetores e fatores de vulnerabilidade (Tese de Doutoramento). Universidade de Lisboa. Disponível em: https://repositorio.ul.pt/handle/10451/28877
Nogueira, M. J., \& Sequeira, C. (2018). A satisfação com a vida académica. Relação com bem-estar e distress psicológico. Revista Portuguesa de Enfermagem de Saúde Mental, Especial 6, 71-76. Doi:10.19131/rpesm.0216

Pulido-Martos, M., Augusto-Landa, J. M., \& LopezZafra, E. (2012). Sources of stress in nursing students: a systematic review of quantitative studies. International Nursing Review, 59(1), 15-25. Doi:10.1111/j.14667657.2011.00939.x

Ribeiro, Í. J. S., Pereira, R., Freire, I. V., de Oliveira, B. G., Casotti, C. A., \& Boery, E. N. (2017). Stress and Quality of Life Among University Students: A Systematic Literature Review. Health Professions Education, 4(2), 70-77. Doi: 10.1016/j.hpe.2017.03.002

Sequeira, C; Carvalho, J; Borges, \& Sousa, C. (2013). Vulnerabilidade mental em estudantes de enfermagem no ensino superior : estudo exploratório. Journal of Nursing and Health, (3), 170-81. Disponível: https://periodicos.ufpel.edu.br/ojs2/index.php/enfermagem/article/ view/3551

Silveira, C., Norton, A., Brandão, I., \& Roma-Torres, A. (2011). Saúde mental em estudantes do ensino superior experiência da consulta de psiquiatria do centro hospitalar São João. Acta Medica Portuguesa, 24(SUPPL.2), 247-256.

Soares, A. M., Pinheiro, M. R., \& Canavarro, J. M. (2015). Transição e adaptação ao ensino superior e a demanda pelo sucesso nas instituições portuguesas. Psychologica, 58(2), 97-116. Doi: 10.14195/1647-8606_58

Soares, A. M., Pereira, A. M., \& Canavarro, J. M. (2015). Promoção da Saúde nas Instituições de Ensino Superior Portuguesas: Reflexões e Desafio. Revista portuguesa de pedagogia, 49(2), 115-137.Doi: 10.14195/1647-8606_58

Van Rooij, E. C. M., Jansen, E. P. W. A., \& van de Grift, W. J. C. M. (2018). First-year university students' academic success: the importance of academic adjustment. European Journal of Psychology of Education, 33(4), 749767. Doi: 10.1007/s10212-017-0347-8 